\title{
POLARIZATION TENSORS OF PLANAR DOMAINS AS FUNCTIONS OF THE ADMITTIVITY CONTRAST
}

\author{
ROLAND GRIESMAIER* AND MARTIN HANKE ${ }^{\dagger}$
}

\begin{abstract}
Electric) polarization tensors describe part of the leading order term of asymptotic voltage perturbations caused by low volume fraction inhomogeneities of the electrical properties of a medium. They depend on the geometry of the support of the inhomogeneities and on their admittivity contrast. Corresponding asymptotic formulas are of particular interest in the design of reconstruction algorithms for determining the locations and the material properties of inhomogeneities inside a body from measurements of current flows and associated voltage potentials on the body's surface. In this work we consider the two-dimensional case only and provide an analytic representation of the polarization tensor in terms of spectral properties of the double layer integral operator associated with the support of simply connected conductivity inhomogeneities. Furthermore, we establish that an (infinitesimal) simply connected inhomogeneity has the shape of an ellipse, if and only if the polarization tensor is a rational function of the admittivity contrast with at most two poles whose residues satisfy a certain algebraic constraint. We also use the analytic representation to provide a proof of the so-called Hashin-Shtrikman bounds for polarization tensors; a similar approach has been taken previously by Golden and Papanicolaou and Kohn and Milton in the context of anisotropic composite materials.
\end{abstract} bounds

Key words. Polarization tensor, Fredholm eigenvalues, shape reconstruction, Hashin-Shtrikman

AMS subject classifications. 35R30, 65N21

Last modified. August 3, 2021

1. Introduction. Electrical impedance tomography is an imaging modality that seeks to recover the electrical conductivity distribution inside a body from measurements of current flows and voltage potentials on its surface. Motivated by numerous potential applications, over the past years a considerable amount of work has been dedicated to use such measurements for the reconstruction of low volume fraction conductivity inhomogeneities inside a known background medium. Efficient reconstruction methods for this problem usually rely on asymptotic representation formulas for the voltage perturbation caused by such anomalies (see, e.g., [5], or Ammari and Kang [2] and the references therein). The relevant leading order term in these asymptotic formulas essentially consists of three parts, namely (i) the gradient of a certain fundamental solution for the background medium, (ii) the gradient of the background potential, and (iii) the (electric) polarization tensor. While the first two components are independent of any inhomogeneities, the polarization tensor fully describes the dependence on the geometry of the support of the inhomogeneity and on its admittivity (i.e., complex conductivity) contrast (see, e.g., Cedio-Fengya, Moskow, and Vogelius [9], or [2]).

In this work we study analytic properties of the polarization tensor as function of the admittivity contrast in a restricted setting: We consider the two-dimensional case only, and we assume that the conductivity distribution of the background medium as well as the conductivity inside the inhomogeneity are constant, and that the support of the conductivity inhomogeneity is a bounded and simply connected Lipschitz domain. Under these assumptions we establish a representation formula for the polarization

\footnotetext{
*Institut für Mathematik, Universität Würzburg, 97074 Würzburg, Germany (roland.griesmaier@uni-wuerzburg.de)

${ }^{\dagger}$ Institut für Mathematik, Johannes Gutenberg-Universität Mainz, 55099 Mainz, Germany (hanke@math.uni-mainz.de)
} 
tensor as function of the admittivity contrast in terms of the spectral decomposition of the double layer integral operator associated with the support of the conductivity inhomogeneity.

This analytic representation of the polarization tensor proves to be a useful tool to analyze certain aspects of the impedance imaging problem with low volume fraction conductivity inhomogeneities. For instance we utilize it in the second part of this work to provide a short and elementary proof of the so-called Hashin-Shtrikman bounds [13] for the trace of polarization tensors and their inverses in terms of the volume of the support of the associated conductivity inhomogeneity, including a discussion of the sharpness of these inequalities, similar to a line of arguments worked out by Golden and Papanicolaou [11] and Kohn and Milton [19] in the framework of anisotropic composites (see also Belyaev and Kozlov [3], Lipton [22], or Capdeboscq and Vogelius [8]). These bounds can, e.g., be used to obtain volume estimates for unkown conductivity inhomogeneities from boundary measurements. We also elaborate on how to extract shape information about the support of an unknown simply connected conductivity inhomogeneity from knowledge of the associated polarization tensor as function of the admittivity contrast. More precisely, we show that the polarization tensor is a rational function of the admittivity contrast with at most two poles and a certain constraint on its residues, if and only if the support of the conductivity inhomogeneity is an ellipse.

We note that data for the polarization tensor corresponding to several admittivity contrasts can, e.g., be acquired using multi-frequency electrical impedance tomography measurements as described in our companion paper [12]. There the analytic representation formula for the polarization tensor to be established below is employed to provide a comprehensive theoretical justification of so-called multi-frequency MUSICtype methods for electrical impedance tomography.

This paper is organized as follows. In the next section we review some facts concerning the mapping properties of layer potential operators associated with the Laplace operator for planar Lipschitz domains. In particular we discuss spectral properties of the double layer integral operator. In Section 3 we use these results to derive the representation formula for polarization tensors as a function of the admittivity contrast; a more explicit version of this formula, which holds under additional smoothness assumptions, is discussed in Section 4. Thereafter we present the two aforementioned applications of these representation formulas: in Section 5 we characterize the polarization tensors corresponding to ellipses as inhomogeneities, and in Section 6 we present the proof of the Hashin-Shtrikman bounds. We conclude with some final remarks.

2. Layer potential operators and their spectra. Let $\Omega \subset \mathbb{R}^{2}$ be a bounded Lipschitz domain, and let $\nu$ be the unit outward normal of $\Omega$, which is well-defined a.e. on $\partial \Omega$. In the sequel we review some classical potential theory for such domains in as much as it is needed here; as a general reference we refer to the monograph by McLean [24].

Denoting the fundamental solution of the two-dimensional Laplacian by

$$
\Phi(x, y)=-\frac{1}{2 \pi} \log |x-y|, \quad x, y \in \mathbb{R}^{2}, x \neq y,
$$

the single and the double layer operators associated with $\partial \Omega$ are defined by

$$
(S \chi)(x)=\int_{\partial \Omega} \Phi(x, y) \chi(y) \mathrm{d} s_{y}, \quad x \in \partial \Omega,
$$


and

$$
(K \varphi)(x)=\int_{\partial \Omega} \partial_{\nu(y)} \Phi(x, y) \varphi(y) \mathrm{d} s_{y}, \quad x \in \partial \Omega,
$$

respectively, where for a.e. $x \in \partial \Omega$ the latter is to be understood in the sense of a Cauchy principal value integral, when the boundary of $\Omega$ lacks smoothness. While the single layer operator belongs to $\mathscr{L}\left(H^{-1 / 2}(\partial \Omega), H^{1 / 2}(\partial \Omega)\right)^{\ddagger}$, the double layer operator is a continuous operator in $\mathscr{L}\left(H^{1 / 2}(\partial \Omega)\right)$.

For $\varphi \in H^{1 / 2}(\partial \Omega)$ we further introduce the double layer potential

$$
u(x)=\int_{\partial \Omega} \partial_{\nu(y)} \Phi(x, y) \varphi(y) \mathrm{d} s_{y}, \quad x \in \Omega,
$$

and the single layer potential

$$
v(x)=\int_{\partial \Omega} \Phi(x, y) \varphi^{\prime}(y) \mathrm{d} s_{y}, \quad x \in \Omega,
$$

with density $\varphi^{\prime}=\partial_{s} \varphi$. Here,

$$
\partial_{s}: H^{1 / 2}(\partial \Omega) \rightarrow H^{-1 / 2}(\partial \Omega)
$$

denotes the derivative operator with respect to arc length along $\partial \Omega$ whose dual operator $\partial_{s}^{\prime}$ with respect to the usual sesqui-linear duality pairing $\langle\cdot, \cdot\rangle$ of $H^{ \pm 1 / 2}(\partial \Omega)$ satisfies $\partial_{s}^{\prime}=-\partial_{s}$. The jump relations of single and double layer potentials imply that the trace of $u$ and the normal derivative of $v$ on $\partial \Omega$ satisfy

$$
\left.u\right|_{\Gamma}=\left(K-\frac{1}{2} I\right) \varphi \quad \text { and } \quad \partial_{\nu} v=\left(K^{\prime}+\frac{1}{2}\right) \varphi^{\prime},
$$

where $K^{\prime} \in \mathscr{L}\left(H^{-1 / 2}(\partial \Omega)\right)$ is the dual operator of $K$.

As established by Verchota [32, Sect. 4] for Lipschitz domains, $v$ is a harmonic conjugate function of $u$, and hence, the above jump relations in combination with the Cauchy-Riemann differential equations imply that

$$
\begin{aligned}
K^{\prime} \varphi^{\prime} & =\left(K^{\prime}+\frac{1}{2} I\right) \varphi^{\prime}-\frac{1}{2} \varphi^{\prime}=\partial_{\nu} v-\frac{1}{2} \varphi^{\prime}=-\left.\partial_{s} u\right|_{\partial \Omega}-\frac{1}{2} \varphi^{\prime} \\
& =-\partial_{s}\left(K-\frac{1}{2} I\right) \varphi-\frac{1}{2} \varphi^{\prime}=-\partial_{s} K \varphi .
\end{aligned}
$$

Likewise we conclude that the hypersingular operator $T \in \mathscr{L}\left(H^{1 / 2}(\partial \Omega), H^{-1 / 2}(\partial \Omega)\right)$ which maps $\varphi \in H^{1 / 2}(\partial \Omega)$ onto the normal derivative of the double layer potential (2.2) satisfies

$$
T \varphi=\partial_{\nu} u=\partial_{s} v=\partial_{s} S \varphi^{\prime}
$$

From these observations we conclude that

$$
K^{\prime} \partial_{s}=-\partial_{s} K \quad \text { and } \quad T=\partial_{s} S \partial_{s},
$$

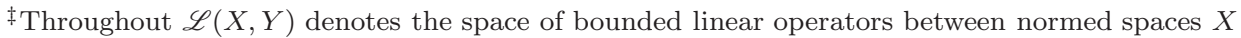
and $Y$, and $\mathscr{L}(X):=\mathscr{L}(X, X)$.
} 
which can subsequently be used to rewrite the fundamental Plemelj identities ([24, p. 244]) in the form

$$
K S=S K^{\prime} \quad \text { and } \quad\left(S \partial_{s}\right)^{2}=K^{2}-\frac{1}{4} I .
$$

Now we turn to the spectral properties of the double layer operator. For smooth domains the spectrum of $K$ is fairly well understood since the work of Plemelj [26]. In this case the operator $K$ is compact, so that its spectrum consists of the origin and a point spectrum, sometimes referred to as Fredholm eigenvalues of the domain $\Omega$. We refer to Schiffer [30] or Khavinson, Putinar, and Shapiro [18] for expositions of the corresponding results. These have recently been extended by Helsing and Perfekt [14] to Lipschitz domains, where $K$ will no longer be compact, in general. While the focus in [14] is primarily on the three-dimensional case, we will develop the corresponding two-dimensional theory below.

At this point we make the additional assumption that $\Omega$ be simply connected and that $\Omega \subset \mathcal{D}$, where $\mathcal{D}$ denotes the unit disk. The first of these two assumptions implies that the trivial eigenvalue $\lambda=-1 / 2$ of $K$ has a one-dimensional eigenspace, which is spanned by the constant functions on $\partial \Omega$. The second assumption, which we remove below when we formulate (and prove) our main result (Theorem 3.3), makes sure that the single layer operator $S$ is selfadjoint and positive as operator in $\mathscr{L}\left(L^{2}(\partial \Omega)\right)$ (cf., e.g., Landkof [21]), and is an isomorphism between $H^{-1 / 2}(\partial \Omega)$ and $H^{1 / 2}(\partial \Omega)$. As such, $S$ has a well-defined square root operator $S^{1 / 2}$ that is an isomorphism in $\mathscr{L}\left(H^{-1 / 2}(\partial \Omega), L^{2}(\partial \Omega)\right)$ and in $\mathscr{L}\left(L^{2}(\partial \Omega), H^{1 / 2}(\partial \Omega)\right)$.

Now we introduce

$$
A=S^{-1 / 2} K S^{1 / 2}
$$

which is a selfadjoint operator in $L^{2}(\partial \Omega)$ by virtue of (2.5). Note that (2.6) is a similarity transformation, hence $A$ and $K$ have the same eigenvalues and spectra, and associated eigenspaces have the same dimensions, respectively. In particular, $\lambda=-1 / 2$ is an eigenvalue of $A$, and its eigenspace is spanned by $S^{-1 / 2} 1$. On the orthogonal complement of this eigenspace the shifted operators $A \pm \frac{1}{2} I$ are contracting (cf., e.g., Steinbach and Wendland [31]). Hence we conclude that the spectrum $\sigma(A)$ of $A$ is contained in

$$
\sigma(A) \subset\{-1 / 2\} \cup[-a, a]
$$

for some $0 \leq a<1 / 2$. Below, we denote by $E$ the spectral decomposition of $A$, i.e., for every Borel set $\omega \subset \mathbb{R}$ there is an orthogonal projection $E(\omega) \in \mathscr{L}\left(L^{2}(\partial \Omega)\right)$ such that

$$
A=\int_{-\infty}^{\infty} \lambda \mathrm{d} E_{\lambda}
$$

(cf., e.g., Rudin [28, Sect. 12.17]).

For smooth domains Plemelj [26] has observed that in two space dimensions the spectra of $A$ and $K$ have rich additional structure due to complex function theory: Namely, the first identity in (2.4) implies that if $\varphi \in H^{1 / 2}(\partial \Omega)$ is an eigenfunction of $K$ for the eigenvalue $\lambda \neq-1 / 2$ then $\varphi^{\prime}$ is an eigenfunction of $K^{\prime}$ for $-\lambda$. (If $\varphi$ is an eigenfunction of $K$ for $\lambda=-1 / 2$ then $\varphi$ is constant and $\varphi^{\prime}$ vanishes.) Since the spectra of $K$ and $K^{\prime}$ are the same it follows that the eigenvalues of $K$ different from 
$\lambda=-1 / 2$ come in pairs that are symmetric with respect to the origin. Concerning the operator $A$ we note that (2.4) and (2.5) imply that

$$
A S^{1 / 2} \partial_{s} S^{1 / 2}=S^{1 / 2} K^{\prime} \partial_{s} S^{1 / 2}=-S^{1 / 2} \partial_{s} K S^{1 / 2}=-S^{1 / 2} \partial_{s} S^{1 / 2} A,
$$

and hence, if $\psi$ is an eigenfunction of $A$ with eigenvalue $\lambda \neq-1 / 2$ then $S^{1 / 2} \partial_{s} S^{1 / 2} \psi$ is an eigenfunction of $A$ for the eigenvalue $-\lambda$.

The analog of Plemelj's result for Lipschitz domains - where $A$ need not have any eigenfunctions besides $S^{-1 / 2} 1$ - is the following result.

Proposition 2.1. Assume that $\Omega \subset \mathcal{D}$ is a simply connected Lipschitz domain. Then for every Borel set $\omega \subset \mathbb{R}$ there holds

$$
E(\omega) S^{1 / 2} \partial_{s} S^{1 / 2}=S^{1 / 2} \partial_{s} S^{1 / 2} E(-\omega) .
$$

Proof. Starting from (2.8) we readily obtain by induction that

$$
A^{n} S^{1 / 2} \partial_{s} S^{1 / 2}=S^{1 / 2} \partial_{s} S^{1 / 2}(-A)^{n} \quad \text { for every } n \geq 0 .
$$

Since

$$
\left\langle A^{n}\left(S^{1 / 2} \partial_{s} S^{1 / 2}\right) \psi, \widetilde{\psi}\right\rangle=\int_{-\infty}^{\infty} \lambda^{n} \mathrm{~d}\left\langle E_{\lambda}\left(S^{1 / 2} \partial_{s} S^{1 / 2}\right) \psi, \widetilde{\psi}\right\rangle
$$

for every $\psi, \widetilde{\psi} \in L^{2}(\partial \Omega)$, and similarly

$$
\begin{aligned}
& \left\langle\left(S^{1 / 2} \partial_{s} S^{1 / 2}\right)(-A)^{n} \psi, \widetilde{\psi}\right\rangle=\left\langle(-A)^{n} \psi,\left(S^{1 / 2} \partial_{s} S^{1 / 2}\right)^{*} \widetilde{\psi}\right\rangle \\
& =\int_{-\infty}^{\infty}(-\lambda)^{n} \mathrm{~d}\left\langle E_{\lambda} \psi,\left(S^{1 / 2} \partial_{s} S^{1 / 2}\right)^{*} \widetilde{\psi}\right\rangle=\int_{-\infty}^{\infty} \lambda^{n} \mathrm{~d}\left\langle E_{-\lambda} \psi,\left(S^{1 / 2} \partial_{s} S^{1 / 2}\right)^{*} \widetilde{\psi}\right\rangle \\
& =\int_{-\infty}^{\infty} \lambda^{n} \mathrm{~d}\left\langle\left(S^{1 / 2} \partial_{s} S^{1 / 2}\right) E_{-\lambda} \psi, \widetilde{\psi}\right\rangle,
\end{aligned}
$$

we find that the measures $\left\langle E(\omega) S^{1 / 2} \partial_{s} S^{1 / 2} \psi, \widetilde{\psi}\right\rangle$ and $\left.\left\langle S^{1 / 2} \partial_{s} S^{1 / 2}\right) E(-\omega) \psi, \widetilde{\psi}\right\rangle$, considered as linear forms on $C(\mathbb{R})$, agree on the space of polynomials. Since $E$ is concentrated on a compact interval, Weierstrass' approximation theorem and Riesz' representation theorem imply that

$$
E(\omega) S^{1 / 2} \partial_{s} S^{1 / 2}=S^{1 / 2} \partial_{s} S^{1 / 2} E(-\omega)
$$

for every Borel set $\omega \subset \mathbb{R}$, which was to be shown.

Corollary 2.2. The set $\sigma(A) \backslash\{-1 / 2\}$ is a compact subset of $(-1 / 2,1 / 2)$ that is symmetric with respect to the origin.

Proof. From (2.7) follows that $\sigma(A) \backslash\{-1 / 2\}$ is a compact subset of $(-1 / 2,1 / 2)$.

Now consider any $\lambda \in \sigma(A) \backslash\{-1 / 2\}$ and any open neighborhood $\omega \subset \mathbb{R}$ of $-\lambda$. Without loss of generality we may assume that $1 / 2 \notin \omega$. Because of $\lambda \in-\omega$ we have $E(-\omega) \neq 0$. Hence there exists $\psi \in L^{2}(\partial \Omega)$ with $\|\psi\|=1$ and $E(-\omega) \psi=\psi$. It thus follows from Proposition 2.1 that

$$
E(\omega) S^{1 / 2} \partial_{s} S^{1 / 2} \psi=S^{1 / 2} \partial_{s} S^{1 / 2} E(-\omega) \psi=S^{1 / 2} \partial_{s} S^{1 / 2} \psi,
$$

and the right-hand side vanishes, if and only if $\psi=c S^{-1 / 2} 1$ for some $c \in \mathbb{R}$, i.e., if and only if $\psi$ is an eigenfunction of $A$ corresponding to $-1 / 2$. The latter, however, cannot be true because the range of $E(-\omega)$ is orthogonal to this eigenspace due to the fact that $-1 / 2 \notin-\omega$. Thus we have established that $E(\omega) \neq 0$ for every sufficiently small neighborhood of $-\lambda$. This proves that $-\lambda \in \sigma(A)$, which was to be shown. 
3. The polarization tensor as a function of the admittivity contrast. We now turn to investigate the polarization tensor of $\Omega$ and derive the main result of this work. Again, for the time being we restrict our attention to Lipschitz domains $\Omega \subset \mathcal{D}$.

Let $x_{1,2}$ and $\nu_{1,2}$ denote the two components of the spatial variable $x \in \mathbb{R}^{2}$ and of the outer unit normal at $x \in \partial \Omega$, respectively. Then the quantities

$$
M_{k l}(\mu ; \Omega)=\int_{\partial \Omega} \nu_{l}(\mu I-K)^{-1} x_{k} \mathrm{~d} s, \quad k, l=1,2,
$$

are well-defined for every $\mu \in \mathbb{C} \backslash \sigma(K)$. They constitute the entries of the (electric) polarization tensor $M(\mu ; \Omega)=\left[M_{k l}(\mu ; \Omega)\right]_{k l} \in \mathbb{C}^{2 \times 2}$ associated with the planar domain $\Omega$ (see [2, Sect. 4.1] for the interpretation of $\mu$ as an admittivity contrast).

Note that

$$
\int_{\partial \Omega} \nu_{l} \mathrm{~d} s=0, \quad l=1,2,
$$

because $\nu_{l}=\nu \cdot \operatorname{grad} x_{l}$, and $x \mapsto x_{l}$ is harmonic in $\Omega$. Furthermore, since $K 1=-1 / 2$, any constant shift of $\Omega$ becomes a constant shift of $(\mu I-K)^{-1} x_{k}$, that cancels in the computation of the polarization tensor. It follows that the polarization tensor is independent of translations of $\Omega$. Finally, given an orthogonal transform $Q \in \mathbb{R}^{2 \times 2}$ and a scaling factor $c>0$, the polarization tensor satisfies

$$
M(\cdot ; c Q(\Omega))=c^{2} Q M(\cdot ; \Omega) Q^{*}
$$

(cf., e.g., [2]).

We rewrite $x_{1}$ as a double layer potential

$$
x_{1}=\int_{\partial \Omega} \partial_{\nu(y)} \Phi(x, y) \varphi(y) \mathrm{d} s_{y}, \quad x \in \Omega,
$$

with density $\varphi \in H^{1 / 2}(\partial \Omega)$, which solves the second kind integral equation

$$
\left(K-\frac{1}{2} I\right) \varphi=\left.x_{1}\right|_{\partial \Omega} .
$$

From (2.4) we conclude that

$$
\left.\nu_{1}\right|_{\partial \Omega}=\partial_{\nu} x_{1}=T \varphi=\partial_{s} S \partial_{s} \varphi
$$

and hence, Plemelj's identities (2.5) yield

$$
\left.\nu_{1}\right|_{\partial \Omega}=S^{-1}\left(S \partial_{s}\right)^{2} \varphi=S^{-1}\left(K^{2}-\frac{1}{4} I\right) \varphi=\left(K^{\prime 2}-\frac{1}{4} I\right) S^{-1} \varphi,
$$

so that

$$
\begin{aligned}
M_{11}(\mu ; \Omega) & =\left\langle S^{-1} \varphi,(\mu I-K)^{-1}\left(K^{2}-\frac{1}{4} I\right)\left(K-\frac{1}{2} I\right) \varphi\right\rangle \\
& =\left\langle S^{-1 / 2} \varphi,(\mu I-A)^{-1}\left(A^{2}-\frac{1}{4} I\right)\left(A-\frac{1}{2} I\right) S^{-1 / 2} \varphi\right\rangle .
\end{aligned}
$$

Using spectral calculus this can be rewritten as

$$
M_{11}(\mu ; \Omega)=\int_{-\infty}^{\infty} \frac{1}{\mu-\lambda} \mathrm{d} \alpha_{\lambda}
$$


where the positive Radon measure $\alpha$ is given by

$$
\alpha(\omega)=\left\|E(\omega)\left(\left(\frac{1}{4} I-A^{2}\right)\left(\frac{1}{2} I-A\right)\right)^{1 / 2} S^{-1 / 2} \varphi\right\|^{2}
$$

for every Borel set $\omega \subset \mathbb{R}$. Formula (3.5) appears in [14] (see also Ammari, Chow, Liu, and Zou [1]), and similar representations of effective conductivity tensors of composite media go back to Bergman [4]; see also [11, 19] and the corresponding Chapter XVIII of the monograph by Milton [25].

The harmonic conjugate $v=x_{2}$ of $u=x_{1}$ in $\Omega$ is specified by (2.3) up to an additive constant, where $\varphi^{\prime}=\partial_{s} \varphi$ again. Accordingly,

$$
\left.\nu_{2}\right|_{\partial \Omega}=\partial_{\nu} x_{2}=\left(K^{\prime}+\frac{1}{2} I\right) \varphi^{\prime},
$$

and hence,

$$
\begin{aligned}
M_{22}(\mu ; \Omega) & =\left\langle\left(K^{\prime}+\frac{1}{2} I\right) \varphi^{\prime},(\mu I-K)^{-1} S \varphi^{\prime}\right\rangle \\
& =\left\langle S^{1 / 2} \varphi^{\prime},\left(A+\frac{1}{2} I\right)(\mu I-A)^{-1} S^{1 / 2} \varphi^{\prime}\right\rangle \\
& =\int_{-\infty}^{\infty} \frac{1}{\mu-\lambda} \mathrm{d} \beta_{\lambda}
\end{aligned}
$$

with

$$
\beta(\omega)=\left\|E(\omega)\left(\frac{1}{2} I+A\right)^{1 / 2} S^{1 / 2} \varphi^{\prime}\right\|^{2}
$$

for every Borel set $\omega \subset \mathbb{R}$. As has been pointed out by Keller [17] the usage of these harmonic conjugates reveals duality properties of these two-dimensional tensors. More precisely, we have the following result.

Lemma 3.1. Let $\Omega \subset \mathcal{D}$ be a simply connected Lipschitz domain. Then

$$
\beta(\omega)=\alpha(-\omega)
$$

for every Borel set $\omega \subset \mathbb{R}$.

Proof. For a given $\omega$ we use Proposition 2.1 and the fact that every function of $A$ commutes with (the projection) $E(\omega)$ to rewrite

$$
\begin{aligned}
\beta(\omega) & =\left\langle E(\omega) S^{1 / 2} \varphi^{\prime},\left(\frac{1}{2} I+A\right) E(\omega) S^{1 / 2} \varphi^{\prime}\right\rangle \\
& =\left\langle S^{1 / 2} \partial_{s} S^{1 / 2} E(-\omega) S^{-1 / 2} \varphi,\left(\frac{1}{2} I+A\right) S^{1 / 2} \partial_{s} S^{1 / 2} E(-\omega) S^{-1 / 2} \varphi\right\rangle .
\end{aligned}
$$

Since $S^{1 / 2}$ is selfadjoint and $\partial_{s}$ is skewadjoint it thus follows from (2.8), (2.4), (2.5), and (2.6) that

$$
\begin{aligned}
\beta(\omega) & =-\left\langle E(-\omega) S^{-1 / 2} \varphi, S^{1 / 2} \partial_{s} S^{1 / 2}\left(\frac{1}{2} I+A\right) S^{1 / 2} \partial_{s} S^{1 / 2} E(-\omega) S^{-1 / 2} \varphi\right\rangle \\
& =-\left\langle E(-\omega) S^{-1 / 2} \varphi,\left(\frac{1}{2} I-A\right) S^{-1 / 2}\left(S \partial_{s}\right)^{2} S^{1 / 2} E(-\omega) S^{-1 / 2} \varphi\right\rangle \\
& =-\left\langle E(-\omega) S^{-1 / 2} \varphi,\left(\frac{1}{2} I-A\right)\left(A^{2}-\frac{1}{4} I\right) E(-\omega) S^{-1 / 2} \varphi\right\rangle .
\end{aligned}
$$


A comparison with (3.6) now yields the assertion.

Making use of this result we can rewrite (3.8) to obtain the formula

$$
M_{22}(\mu ; \Omega)=\int_{-\infty}^{\infty} \frac{1}{\mu+\lambda} \mathrm{d} \alpha_{\lambda}=-M_{11}(-\mu ; \Omega),
$$

see [17] again. Next we consider the off-diagonal entries of the polarization tensor. Using (3.3) and (3.7) there holds

$$
\begin{aligned}
M_{12}(\mu ; \Omega) & =\left\langle\left(K^{\prime}+\frac{1}{2} I\right) \varphi^{\prime},(\mu I-K)^{-1}\left(K-\frac{1}{2} I\right) \varphi\right\rangle \\
& =\left\langle S^{1 / 2} \varphi^{\prime},(\mu I-A)^{-1}\left(A^{2}-\frac{1}{4} I\right) S^{-1 / 2} \varphi\right\rangle,
\end{aligned}
$$

and hence,

$$
M_{12}(\mu ; \Omega)=\int_{-\infty}^{\infty} \frac{1}{\mu-\lambda} \mathrm{d} \gamma_{\lambda}
$$

with

$$
\gamma(\omega)=\left\langle S^{1 / 2} \varphi^{\prime}, E(\omega)\left(A^{2}-\frac{1}{4} I\right) S^{-1 / 2} \varphi\right\rangle
$$

for every Borel set $\omega \subset \mathbb{R}$. Employing (3.4) it is straightforward to deduce the same representation for $M_{21}(\mu ; \Omega)$. We mention, though, that it is well-known that the polarization tensor is symmetric (see, e.g., [2]).

A similar argument as in Lemma 3.1 reveals that the measure $\gamma$ is antisymmetric:

Lemma 3.2. Let $\Omega \subset \mathcal{D}$ be a simply connected Lipschitz domain. Then

$$
\gamma(\omega)=-\gamma(-\omega)
$$

for every Borel set $\omega \subset \mathbb{R}$. Moreover,

$$
|\gamma(\omega)| \leq(\alpha(\omega) \alpha(-\omega))^{1 / 2} \leq \alpha^{\mathrm{symm}}(\omega):=\frac{1}{2}(\alpha(\omega)+\alpha(-\omega)) .
$$

Proof. Using Proposition 2.1, (2.8), and (3.13), we find that for any Borel set $\omega \subset \mathbb{R}$ there holds

$$
\begin{aligned}
\gamma(\omega) & =\left\langle E(\omega) S^{1 / 2} \varphi^{\prime},\left(A^{2}-\frac{1}{4} I\right) E(\omega) S^{-1 / 2} \varphi\right\rangle \\
& =\left\langle S^{1 / 2} \partial_{s} S^{1 / 2} E(-\omega) S^{-1 / 2} \varphi,\left(A^{2}-\frac{1}{4}\right) E(\omega) S^{-1 / 2} \varphi\right\rangle \\
& =-\left\langle E(-\omega) S^{-1 / 2} \varphi, S^{1 / 2} \partial_{s} S^{1 / 2}\left(A^{2}-\frac{1}{4} I\right) E(\omega) S^{-1 / 2} \varphi\right\rangle \\
& =-\left\langle E(-\omega) S^{-1 / 2} \varphi,\left(A^{2}-\frac{1}{4} I\right) S^{1 / 2} \partial_{s} S^{1 / 2} E(\omega) S^{-1 / 2} \varphi\right\rangle \\
& =-\left\langle E(-\omega) S^{-1 / 2} \varphi,\left(A^{2}-\frac{1}{4} I\right) E(-\omega) S^{1 / 2} \varphi^{\prime}\right\rangle \\
& =-\gamma(-\omega) .
\end{aligned}
$$


Concerning the second assertion we apply the Cauchy-Schwarz inequality to estimate

$$
\begin{aligned}
|\gamma(\omega)| & =\left|\left\langle E(\omega)\left(\frac{1}{2} I+A\right)^{1 / 2} S^{1 / 2} \varphi^{\prime}, E(\omega)\left(\frac{1}{2} I+A\right)^{1 / 2}\left(\frac{1}{2} I-A\right) S^{-1 / 2} \varphi\right\rangle\right| \\
& \leq(\alpha(\omega) \beta(\omega))^{1 / 2} \leq \frac{1}{2}(\alpha(\omega)+\beta(\omega)) .
\end{aligned}
$$

A final application of Lemma 3.1 yields the desired inequality (3.14).

We are now ready to state our main result, for which we drop the assumption that $\Omega \subset \mathcal{D}$.

TheOREM 3.3. Let $\Omega \subset \mathbb{R}^{2}$ be a bounded simply connected Lipschitz domain. Then the polarization tensor $M(\mu ; \Omega)$ from (3.1) is of the form

$$
M(\mu ; \Omega)=\left[\begin{array}{cc}
\int_{-\infty}^{\infty} \frac{r^{2}(\lambda)}{\mu-\lambda} \mathrm{d} \alpha_{\lambda}^{\mathrm{symm}} & \int_{-\infty}^{\infty} \frac{c(\lambda) r(\lambda) r(-\lambda)}{\mu-\lambda} \mathrm{d} \alpha_{\lambda}^{\mathrm{symm}} \\
\int_{-\infty}^{\infty} \frac{c(\lambda) r(\lambda) r(-\lambda)}{\mu-\lambda} \mathrm{d} \alpha_{\lambda}^{\mathrm{symm}} & \int_{-\infty}^{\infty} \frac{r^{2}(-\lambda)}{\mu-\lambda} \mathrm{d} \alpha_{\lambda}^{\mathrm{symm}}
\end{array}\right]
$$

for $\mu \in \mathbb{C} \backslash \sigma(K)$, where $\alpha^{\text {symm }}$ is a positive symmetric Radon measure supported on $\sigma(K) \backslash\{-1 / 2\}$, which is a compact subset of $(-1 / 2,1 / 2), r$ is a nonnegative $L^{\infty}\left(\mathbb{R} ; \alpha^{\text {symm }}\right)$ function and $c$ is an odd $d^{*}$ and real valued $L^{\infty}\left(\mathbb{R} ; \alpha^{\text {symm }}\right)$ function with $|c| \leq 1$. Moreover, there holds

$$
\int_{-\infty}^{\infty} r^{2}(\lambda) \mathrm{d} \alpha_{\lambda}^{\text {symm }}=\lim _{\mu \rightarrow \infty} \mu M_{k k}(\mu ; \Omega)=|\Omega|
$$

for $k=1,2$.

Proof. Assume first that $\Omega \subset \mathcal{D}$, so that the previous results apply, and define $\alpha, \beta$, and $\gamma$ as in (3.6), (3.9), and (3.13), respectively, where $\varphi \in H^{1 / 2}(\partial \Omega)$ is the unique solution of (3.3). Then, by virtue of Lemma 3.2 there holds $\gamma \ll \alpha^{\text {symm }}$, while $\alpha \ll \alpha^{\text {symm }}$ is a trivial statement. Accordingly the Radon-Nikodym Theorem (cf., e.g., Evans and Gariepy [10, p. 40]) implies the existence of functions $h, \widetilde{r} \in L^{1}\left(\mathbb{R} ; \alpha^{\mathrm{symm}}\right)$ such that

$$
\gamma(\omega)=\int_{\omega} h(\lambda) \mathrm{d} \alpha_{\lambda}^{\mathrm{symm}} \quad \text { and } \quad \alpha(\omega)=\int_{\omega} \widetilde{r}(\lambda) \mathrm{d} \alpha_{\lambda}^{\mathrm{symm}}
$$

for every Borel set $\omega \subset \mathbb{R}$. A localization argument (cf., e.g., [10, p. 43]) shows that we can choose $\widetilde{r}$ to satisfy

$$
0 \leq \widetilde{r}(\lambda) \leq 2, \quad \lambda \in \mathbb{R}
$$

and hence we can rewrite $\widetilde{r}=r^{2}$ for some nonnegative $r \in L^{\infty}\left(\mathbb{R} ; \alpha^{\text {symm }}\right)$. Similarly, we find from Lemma 3.2 that $h \in L^{\infty}\left(\mathbb{R} ; \alpha^{\text {symm }}\right)$ can be chosen to satisfy $h(\lambda)=$ $-h(-\lambda)$ for $\alpha^{\text {symm }}$ a.e. $\lambda \in \mathbb{R}$, and

$$
|h(\lambda)| \leq(\widetilde{r}(\lambda) \widetilde{r}(-\lambda))^{1 / 2}=r(\lambda) r(-\lambda), \quad \lambda \in \mathbb{R} .
$$

\footnotetext{
${ }^{*}$ Here, odd means that $c(\lambda)=-c(-\lambda)$ for $\alpha^{\text {symm }}$ a.e. $\lambda \in \mathbb{R}$; in particular, $c$ can be chosen to satisfy $c(0)=0$.
} 
Thus the odd function

$$
c(\lambda):=\left\{\begin{array}{cl}
\frac{h(\lambda)}{r(\lambda) r(-\lambda)} & \text { if } r(\lambda) r(-\lambda) \neq 0 \\
1 & \text { else }
\end{array}\right.
$$

is Borel measurable on $\mathbb{R}$ and satisfies $|c| \leq 1$. Now the asserted representation of $M(\mu ; \Omega)$ follows from (3.5), (3.10), and (3.12).

Concerning the support of $\alpha^{\text {symm }}$ we first recall from Corollary 2.2 that $\sigma(K) \backslash$ $\{-1 / 2\}=\sigma(A) \backslash\{-1 / 2\}$ is a compact subset of $(-1 / 2,1 / 2)$ that is symmetric with respect to the origin. Moreover, from

$$
\left\langle S^{1 / 2} \varphi^{\prime}, S^{-1 / 2} 1\right\rangle=\left\langle\varphi^{\prime}, 1\right\rangle=0
$$

we conclude that $S^{1 / 2} \varphi^{\prime}$ is orthogonal to the eigenspace of $A$ corresponding to the eigenvalue $\lambda=-1 / 2$. Hence, it follows from (3.9) that $\beta$ is supported in $\sigma(A) \backslash\{-1 / 2\}$, and the same must be true for $\alpha^{\text {symm }}$ due to Lemma 3.1 and the definition of $\alpha^{\text {symm }}$ in (3.14). This proves the representation formula for $M(\mu ; \Omega)$ under the additional assumption that $\Omega \subset \mathcal{D}$.

For a general domain we choose $\varepsilon>0$ sufficiently small so that $\varepsilon \Omega \subset \mathcal{D}$. Making use of (3.2), the above result (with measure $\alpha^{\text {symm }}$ ) for $\varepsilon \Omega$ provides the corresponding result for $\Omega$, with $\alpha^{\mathrm{symm}}$ replaced by $\alpha^{\mathrm{symm}} / \varepsilon^{2}$. Note that by virtue of (2.1) the spectrum of $K$ is independent of rescalings of the domain.

Finally, while the first equality in (3.15) is an immediate consequence of the representation of the diagonal entries of the polarization tensor, the original definition (3.1) of $M_{k k}(\mu ; \Omega)$ and Green's formula yield

$$
\begin{aligned}
\mu M_{k k}(\mu ; \Omega) & =\int_{\partial \Omega} \nu_{k}\left(I-\frac{1}{\mu} K\right)^{-1} x_{k} \mathrm{~d} s \\
& \longrightarrow \int_{\partial \Omega} \nu_{k} x_{k} \mathrm{~d} s=\int_{\Omega}\left|\nabla x_{k}\right|^{2} \mathrm{~d} x=|\Omega|
\end{aligned}
$$

for $k=1,2$ as $\mu \rightarrow \infty$, and hence the second identity in (3.15) follows.

We mention that (3.15) is well known (cf., e.g., [14]).

REMARK 3.4. According to Theorem $3.3 M(\mu ; \Omega)$ extends as an analytic function to $\mathbb{C} \backslash[-a, a]$ with $0 \leq a<1 / 2$ as in (2.7), and this extension coincides with the original definition (3.1) for $\mu \neq-1 / 2$. The extension to $\mu=-1 / 2$ satisfies the alternative (dual) representation

$$
M_{k l}(\mu ; \Omega)=\int_{\partial \Omega} x_{k}\left(\mu I-K_{\diamond}^{\prime}\right)^{-1} \nu_{l} \mathrm{~d} s, \quad k, l=1,2,
$$

in which $K_{\diamond}^{\prime}$ denotes the restriction of $K^{\prime}$ to its invariant subspace

$$
L_{\diamond}^{2}(\partial \Omega)=\left\{\chi \in L^{2}(\partial \Omega): \int_{\partial \Omega} \chi \mathrm{d} s=0\right\},
$$

on which $\frac{1}{2} I-K_{\diamond}^{\prime}$ is invertible (cf., e.g., [2]).

4. The polarization tensor of smooth planar domains. When the double layer operator $K: H^{1 / 2}(\partial \Omega) \rightarrow H^{1 / 2}(\partial \Omega)$ is compact, for example, if $\Omega$ is a $C^{2}$ 
domain (cf. Kress [20]), the representation of Theorem 3.3 of the polarization tensor simplifies to

$$
M(\mu ; \Omega)=\left[\begin{array}{cc}
\sum_{n \in \mathbb{Z}} \frac{r_{n}^{2}}{\mu-\lambda_{n}} & \sum_{n \in \mathbb{Z}} \frac{c_{n} r_{n} r_{-n}}{\mu-\lambda_{n}} \\
\sum_{n \in \mathbb{Z}} \frac{c_{n} r_{n} r_{-n}}{\mu-\lambda_{n}} & \sum_{n \in \mathbb{Z}} \frac{r_{-n}^{2}}{\mu-\lambda_{n}}
\end{array}\right],
$$

where $\left\{\lambda_{n}: n \in \mathbb{N}\right\}$ is an enumeration of the nonnegative eigenvalues of $K$ (ignoring multiplicities), $\lambda_{-n}=-\lambda_{n}$ for $n \in \mathbb{N}$, and $\lambda_{0}=0$. In the notation of Theorem 3.3 the nonnegative coefficients $r_{ \pm n}$ and the real coefficients $c_{n}=-c_{-n}$ with $\left|c_{n}\right| \leq 1$ are given by

$$
r_{n}=r\left(\lambda_{n}\right) \alpha^{\mathrm{symm}}\left(\left\{\lambda_{n}\right\}\right) \quad \text { and } \quad c_{n}=c\left(\lambda_{n}\right)
$$

for $n \in \mathbb{Z}$. Note that if zero does not happen to be an eigenvalue of $A$ then there holds $r_{0}=0$; note further that $c_{0}=0$, independent of whether $\lambda=0$ is an eigenvalue of $A$, or not.

We conclude that in the smooth case the polarization tensor $M(\mu ; \Omega)$ is a meromorphic function of $\mu \in \widehat{\mathbb{C}} \backslash\{0\}$ into the space of complex symmetric $2 \times 2$ matrices, with simple poles at eigenvalues $\lambda \notin\{-1 / 2,0\}$ of the double layer operator $K$, and with limiting value zero at infinity. Here, as usual, $\widehat{\mathbb{C}}=\mathbb{C} \cup\{\infty\}$ denotes the extended complex plane.

Of course, (4.1) is much more easy to derive in the smooth case by expanding

$$
S^{-1 / 2} \varphi=\sum_{n \in \mathbb{Z}} r_{n} \psi_{n}
$$

where $\psi_{n} \in L^{2}(\partial \Omega), n \in \mathbb{Z}$, are eigenfunctions of $A$ for the eigenvalues $\lambda_{n}$, respectively, normalized to satisfy

$$
\left\|\psi_{n}\right\|^{2}=\left(\left(\frac{1}{2}-\lambda_{n}\right)\left(\frac{1}{4}-\lambda_{n}^{2}\right)\right)^{-1}, \quad n \in \mathbb{Z} .
$$

Then there holds

$$
S^{1 / 2} \varphi^{\prime}=\left(S^{1 / 2} \partial_{s} S^{1 / 2}\right) S^{-1 / 2} \varphi=\sum_{n \in \mathbb{Z}} r_{n} \widetilde{\psi}_{n}
$$

where $\widetilde{\psi}_{n}=S^{1 / 2} \partial_{s} S^{1 / 2} \psi_{n} \in L^{2}(\partial \Omega)$ are eigenfunctions of $A$ corresponding to $-\lambda_{n}=$ $\lambda_{-n}$ (cf. (2.8)), and

$$
\begin{aligned}
\left\|\widetilde{\psi}_{n}\right\|^{2} & =\left\|S^{1 / 2} \partial_{s} S^{1 / 2} \psi_{n}\right\|^{2}=-\left\langle\psi_{n}, S^{-1 / 2}\left(S \partial_{s}\right)^{2} S^{1 / 2} \psi_{n}\right\rangle \\
& =-\left\langle\psi_{n},\left(A^{2}-\frac{1}{4} I\right) \psi_{n}\right\rangle=\left(\frac{1}{4}-\lambda_{n}^{2}\right)\left\|\psi_{n}\right\|^{2}=\left(\frac{1}{2}-\lambda_{n}\right)^{-1} .
\end{aligned}
$$

This sheds some additional light on the parameters $c_{n}$ in (4.1): From (3.11) and the fact that eigenspaces of $A$ corresponding to different eigenvalues are mutually orthogonal we deduce that

$$
\begin{aligned}
M_{12}(\mu ; \Omega) & =\sum_{n \in \mathbb{Z}} r_{-n} r_{n} \frac{1}{\mu-\lambda_{n}}\left(\lambda_{n}^{2}-\frac{1}{4}\right)\left\langle\widetilde{\psi}_{-n}, \psi_{n}\right\rangle \\
& =-\sum_{n \in \mathbb{Z}} \frac{r_{n} r_{-n}}{\mu-\lambda_{n}} \frac{\left\langle\widetilde{\psi}_{-n}, \psi_{n}\right\rangle}{\left\|\widetilde{\psi}_{-n}\right\|\left\|\psi_{n}\right\|} .
\end{aligned}
$$


It follows that

$$
c_{n}=-\frac{\left\langle\widetilde{\psi}_{-n}, \psi_{n}\right\rangle}{\left\|\widetilde{\psi}_{-n}\right\|\left\|\psi_{n}\right\|}
$$

is the cosine of the angle between $\psi_{n}$ and $-\widetilde{\psi}_{-n}$, both of which are eigenfunctions of $A$ corresponding to the same eigenvalue $\lambda_{n}$. In particular, if all eigenspaces of $A$ have dimension one then $c_{n}= \pm 1$ for every $n \in \mathbb{Z}$. In general, however, the coefficients $c_{n}$ can take values different from \pm 1 as the following example shows.

ExAmple 4.1. A domain $\Omega \subset \mathbb{R}^{2}$ shall be called cyclic with index $k \geq 2$, if $Q(\Omega)=\Omega$ where $Q$ denotes the rotation by $2 \pi / k$ (clockwise, or counter-clockwise). By virtue of (3.2) there holds

$$
M(\mu ; \Omega)=Q M(\mu ; \Omega) Q^{*}
$$

for every cyclic domain $\Omega$, and hence, if $p$ is an eigenvector of the polarization tensor for the eigenvalue $\zeta$ then $Q p$ is another eigenvector for the same eigenvalue. Since $p$ and $Q p$ are linearly independent whenever $k \geq 3$, it thus follows that

$$
M(\mu ; \Omega)=f(\mu) I,
$$

where $f$ is a scalar complex function of $\mu$, and $I$ is the $2 \times 2$ identity matrix. Compare, e.g., [2, p. 102], for the same argument.

In particular, if $\Omega$ is a $C^{2}$ domain that is cyclic with index $k \geq 3$ then

$$
f(\mu)=\sum_{n \in \mathbb{Z}} \frac{r_{n}^{2}}{\mu-\lambda_{n}}
$$

is a scalar meromorphic function of $\mu \in \widehat{\mathbb{C}} \backslash\{0\}$ by virtue of (4.1). A further comparison with (4.1) yields that in this case we must have $r_{n}=r_{-n}$, and hence, that $c_{n}=0$ for every pole $\lambda_{n}$ occuring in the representation formula.

Thus, it follows from (4.2) and the discussion following it that every Fredholm eigenvalue $\lambda_{n}$ that is present in (4.1) must have multiplicity greater than one, and that the two eigenfunctions $\psi_{n}$ and $\widetilde{\psi}_{-n}$ occuring in (4.2) must be orthogonal to each other. In fact, under the given assumptions one can represent the double layer integral operator as a block circulant integral operator, and use this representation to convince oneself that for such domains $K$ has many eigenvalues of higher multiplicities, and that the corresponding eigenfunctions in (4.2) are, indeed, orthogonal.

5. Polarization tensors with no more than two poles. We want to use Theorem 3.3 to completely characterize all bounded and simply connected Lipschitz domains $\Omega \subset \mathbb{R}^{2}$ for which the polarization tensor happens to be a rational function of the entire complex plane with at most two poles, and with coefficients $c_{n}$ in (4.1) restricted to have absolute values equal to one. In other words, we assume that

$$
M(\mu ; \Omega)=\left[\begin{array}{cc}
\frac{r_{+}^{2}}{\mu-\lambda}+\frac{r_{-}^{2}}{\mu+\lambda} & \frac{r_{+} r_{-}}{\mu-\lambda}-\frac{r_{+} r_{-}}{\mu+\lambda} \\
\frac{r_{+} r_{-}}{\mu-\lambda}-\frac{r_{+} r_{-}}{\mu+\lambda} & \frac{r_{-}^{2}}{\mu-\lambda}+\frac{r_{+}^{2}}{\mu+\lambda}
\end{array}\right],
$$


where $r_{ \pm} \geq 0$, (at most) one of which may be zero, and $\pm \lambda \in(-1 / 2,1 / 2)$. Note that $M(\mu ; \Omega)$ either has two poles if $\lambda \neq 0$ or one pole when $\lambda=0$.

Choosing

$$
c=\frac{r_{+}}{\left(r_{+}^{2}+r_{-}^{2}\right)^{1 / 2}} \quad \text { and } \quad s=\frac{r_{-}}{\left(r_{+}^{2}+r_{-}^{2}\right)^{1 / 2}}
$$

we obtain

$$
\left[\begin{array}{ll}
c & s
\end{array}\right] M(\mu ; \Omega)\left[\begin{array}{l}
c \\
s
\end{array}\right]=\frac{r_{+}^{4}+2 r_{+}^{2} r_{-}^{2}+r_{-}^{4}}{r_{+}^{2}+r_{-}^{2}} \frac{1}{\mu-\lambda}=\frac{r_{+}^{2}+r_{-}^{2}}{\mu-\lambda},
$$

and — by virtue of (3.1) — the left hand side equals

$$
\int_{\partial \Omega} \partial_{\nu} u^{-}(\mu I-K)^{-1} u^{-} \mathrm{d} s \quad \text { for } \quad u^{-}(x)=c x_{1}+s x_{2} .
$$

Rewriting $u^{-}$in $\Omega$ as a double layer potential with density $\varphi$, we conclude as in (3.5) that $S^{-1 / 2} \varphi$ is an eigenfunction of $A$ for the eigenvalue $\lambda$, and hence, $\varphi$ is an eigenfunction of the double layer operator $K$ for the same eigenvalue.

It follows from the jump relations that the double layer potential

$$
\int_{\partial \Omega} \partial_{\nu(y)} \Phi(x, y) \varphi(y) \mathrm{d} s(y)= \begin{cases}u^{-}(x)=c x_{1}+s x_{2}, & x \in \Omega, \\ u^{+}(x), & x \in \mathbb{R}^{2} \backslash \bar{\Omega},\end{cases}
$$

satisfies

$$
\left.u^{+}\right|_{\partial \Omega}=\left(K+\frac{1}{2} I\right) \varphi=\frac{2 \lambda+1}{2} \varphi=\left.\frac{2 \lambda+1}{2 \lambda-1} u^{-}\right|_{\partial \Omega} .
$$

Thus,

$$
\widetilde{u}(x)= \begin{cases}u^{-}(x), & x \in \Omega, \\ u^{+}(x)+\frac{2}{1-2 \lambda}\left(c x_{1}+s x_{2}\right), & x \in \mathbb{R}^{2} \backslash \Omega,\end{cases}
$$

solves the transmission problem

$$
\begin{array}{rlrl}
\Delta \widetilde{u} & =0 & & \text { in } \mathbb{R}^{2} \backslash \partial \Omega, \\
\left.\widetilde{u}\right|_{\partial \Omega} ^{+}=\left.\widetilde{u}\right|_{\partial \Omega} ^{-},\left.\quad \partial_{\nu} \widetilde{u}\right|_{\partial \Omega} ^{+}=\left.\frac{3-2 \lambda}{1-2 \lambda} \partial_{\nu} \widetilde{u}\right|_{\partial \Omega} ^{-} & & \text {on } \partial \Omega, \\
\widetilde{u}(x) & -\frac{2}{1-2 \lambda}\left(c x_{1}+s x_{2}\right) \longrightarrow 0 & & \text { as }|x| \rightarrow \infty .
\end{array}
$$

Since the gradient of $\widetilde{u}$ also happens to be constant in $\Omega$, it follows from a variant of the strong Eshelby conjecture, which was proved by Ru and Schiavone [27] (see also Kang and Milton [16], and Liu [23]), that $\Omega$ is an ellipse.

In the following we give a slightly extended version of the proof from [27] to show that this ellipse $\Omega$ is indeed completely determined up to translations by knowing the full polarization tensor for all admittivity contrasts; in fact, due to symmetry and (3.10) it is sufficient to know the first column of $M(\mu ; \Omega)$.

Given $u^{ \pm}$and $\varphi$ as above, we consider the restrictions $v^{ \pm}$of the associated single layer potential $v$ of (2.3) to $\mathbb{R}^{2} \backslash \bar{\Omega}$ and $\Omega$, respectively, which provide harmonic conjugates of $u^{ \pm}$with $v^{+}(x) \rightarrow 0$ for $|x| \rightarrow \infty$. Accordingly,

$$
v^{-}(x)=c x_{2}-s x_{1}+d, \quad x \in \Omega,
$$


where $d \in \mathbb{R}$ is a constant, and hence, $v^{+}$has boundary values

$$
v^{+}(x)=v^{-}(x)=c x_{2}-s x_{1}+d, \quad x \in \partial \Omega .
$$

By the Riemann mapping theorem there is a unique conformal transformation $\Psi$ that takes the exterior of the unit disk $\mathcal{D} \subset \widehat{\mathbb{C}}$ onto $\widehat{\mathbb{C}} \backslash \bar{\Omega}$, and satisfies

$$
\Psi(\infty)=\infty, \quad \gamma=\Psi^{\prime}(\infty)>0 .
$$

The parameter $\gamma$ is called the capacity of $\Omega . \Psi$ has a Laurent expansion of the form

$$
\Psi(\zeta)=\gamma \zeta+\sum_{k=1}^{\infty} a_{k} \zeta^{-k}, \quad|\zeta| \geq 1,
$$

with coefficients $a_{k} \in \mathbb{C}, k \in \mathbb{N}$; note that we can assume without loss of generality that no zero order term occurs, since the exact position of $\Omega$ does not enter into the polarization tensor.

Consider now the analytic function

$$
F(z)=u^{+}(z)+\mathrm{i} v^{+}(z), \quad z \in \widehat{\mathbb{C}} \backslash \Omega,
$$

which is bounded and satisfies $F(z) \rightarrow 0$ for $z \rightarrow \infty$. By virtue of (5.2) and (5.3) its boundary values are

$$
F(z)=-q \operatorname{Re}\left(e^{-\mathrm{i} \phi} z\right)+\mathrm{i} \operatorname{Im}\left(e^{-\mathrm{i} \phi} z\right)+\mathrm{i} d=\frac{1-q}{2} e^{-\mathrm{i} \phi} z-\frac{1+q}{2} e^{\mathrm{i} \phi} \bar{z}+\mathrm{i} d
$$

on $\partial \Omega$, where

$$
q=\frac{1+2 \lambda}{1-2 \lambda}>0 \quad \text { and } \quad e^{\mathrm{i} \phi}=c+\mathrm{i} s .
$$

It follows that $G=F \circ \Psi$ is a bounded analytic function in $\widehat{\mathbb{C}} \backslash \mathcal{D}$ with a zero at infinity and limiting values

$$
G\left(e^{\mathrm{i} \theta}\right)=\frac{1-q}{2} e^{-\mathrm{i} \phi} \Psi\left(e^{\mathrm{i} \theta}\right)-\frac{1+q}{2} e^{\mathrm{i} \phi} \overline{\Psi\left(e^{\mathrm{i} \theta}\right)}+\mathrm{i} d, \quad 0 \leq \theta<2 \pi,
$$

on $\partial \mathcal{D}$. Inserting the Laurent series (5.4) of $\Psi$ we obtain

$$
\begin{aligned}
G\left(e^{\mathrm{i} \theta}\right)=-\sum_{k=2}^{\infty} & \frac{1+q}{2} e^{\mathrm{i} \phi} \overline{a_{k}} e^{\mathrm{i} k \theta}+\left(\frac{1-q}{2} e^{-\mathrm{i} \phi} \gamma-\frac{1+q}{2} e^{\mathrm{i} \phi} \overline{a_{1}}\right) e^{\mathrm{i} \theta}+\mathrm{i} d \\
+ & \left(\frac{1-q}{2} e^{-\mathrm{i} \phi} a_{1}-\frac{1+q}{2} e^{\mathrm{i} \phi} \gamma\right) e^{-\mathrm{i} \theta}+\sum_{k=2}^{\infty} \frac{1-q}{2} e^{-\mathrm{i} \phi} a_{k} e^{-\mathrm{i} k \theta} .
\end{aligned}
$$

On the other hand, since $G: \widehat{\mathbb{C}} \backslash \mathcal{D} \rightarrow \mathbb{C}$ is a bounded analytic function with $G(\infty)=0$, its Laurent series has the form

$$
G(\zeta)=\sum_{k=1}^{\infty} b_{k} \zeta^{-k}
$$

and inserting $\zeta=e^{\mathrm{i} \theta}$ and comparing the result with (5.5) it follows that

$$
\frac{1+q}{2} e^{\mathrm{i} \phi} \overline{a_{k}}=0 \quad \text { for all } k \geq 2,
$$


and

$$
\frac{1-q}{2} e^{-\mathrm{i} \phi} \gamma-\frac{1+q}{2} e^{\mathrm{i} \phi} \overline{a_{1}}=0 .
$$

Since $q>0$ this implies that $a_{k}=0$ for all $k \geq 2$, and that

$$
a_{1}=\frac{1-q}{1+q} e^{\mathrm{i} 2 \phi} \gamma=-2 \lambda e^{\mathrm{i} 2 \phi} \gamma
$$

i.e.,

$$
\Psi(\zeta)=\gamma e^{\mathrm{i} \phi}\left(\left(e^{-\mathrm{i} \phi} \zeta\right)-2 \lambda\left(e^{-\mathrm{i} \phi} \zeta\right)^{-1}\right) .
$$

This shows that $\Psi$ is a Joukowski transformation which takes the unit circle onto an ellipse with eccentricity $\max \{q, 1 / q\}$ (resp. a disk, when $\lambda=0$ ) centered at the origin, whose axes have polar angles $\phi$ and $\phi+\pi / 2$. Hence, $\Omega$ is the interior of this ellipse, the volume of which is $|\Omega|=r_{+}^{2}+r_{-}^{2}$ by virtue of (3.15).

We summarize our findings in the following theorem.

THEOREM 5.1. If $M(\mu ; \Omega)$ is given by (5.1) then $\Omega$ is an ellipse whose half axes of lengths

$$
a=\frac{\left(r_{+}^{2}+r_{-}^{2}\right)^{1 / 2}}{\sqrt{\pi}}\left(\frac{1-2 \lambda}{1+2 \lambda}\right)^{1 / 2} \quad \text { and } \quad b=\frac{\left(r_{+}^{2}+r_{-}^{2}\right)^{1 / 2}}{\sqrt{\pi}}\left(\frac{1+2 \lambda}{1-2 \lambda}\right)^{1 / 2}
$$

make angles $\phi=\arctan \left(r_{-} / r_{+}\right)$and $\phi+\pi / 2$ to the horizontal axis, respectively.

REMARK 5.2. In the literature (e.g., in [2, Sect. 4.11.1]) the polarization tensor of an unknown domain $\Omega$ for a fixed admittivity contrast $\mu$ is sometimes used to determine a so-called equivalent ellipse $\mathcal{E}$ that shares this particular polarization tensor. It follows from the above derivation that this ellipse, in fact, changes with $\mu$-unless $\Omega$ is an ellipse, in which case $\Omega=\mathcal{E}$.

For, if the "equivalent ellipse" would not change with $\mu$, (say, taken from a countable set $\left\{\mu_{k}\right\}$ that clusters somewhere in $\left.\widehat{\mathbb{C}} \backslash(-1 / 2,1 / 2)\right)$ then the polarization tensors of $\mathcal{E}$ and $\Omega$ would coincide for these arguments and by the uniqueness theorem for analytic functions the polarization tensor of $\Omega$ would be of the same form (5.1) as the one of $\mathcal{E}$. As we have proved, this implies $\Omega=\mathcal{E}$.

REMARK 5.3. Given the result of this section one might ask oneself whether the knowledge of the polarization tensor $M(\mu ; \Omega)$ as a function of the admittivity contrast suffices to determine the shape of a general simply connected and bounded Lipschitz domain $\Omega$. In fact, the best possible result to expect is that this information determines $\Omega$ up to reflections, because $M(\mu ;-\Omega)=M(\mu ; \Omega)$ by virtue of (3.2).

In this context the authors of [1] numerically optimize the shape of a target domain to fit the eigenvalues of the corresponding double layer operator $K$ to the poles of a given polarization tensor. Since these poles only constitute a subset of the eigenvalues of $K$, in general (see the ellipse as an example), this can be a very underdetermined problem to solve. Moreover, Schiffer [29] has proved that the eigenvalues of $K$ are invariant under Möbius transformations of $\partial \Omega$; therefore knowledge of the poles alone does not suffice to completely determine $\Omega$.

6. The isoperimetric inequalities. In this section we revisit (in two space dimensions) the so-called isoperimetric inequalities, also known as Hashin-Shtrikman 
bounds,

$$
\begin{gathered}
\frac{2}{|\mu|}|\Omega| \leq|\operatorname{Tr}(M(\mu ; \Omega))|<\frac{8|\mu|}{4 \mu^{2}-1}|\Omega|, \\
\left|\operatorname{Tr}\left(M(\mu ; \Omega)^{-1}\right)\right| \leq \frac{2|\mu|}{|\Omega|},
\end{gathered}
$$

valid for $\mu \in \mathbb{R} \backslash(-1 / 2,1 / 2)$. We refer to [2] and [25] for the background of these inequalities, and for variational proofs of them (see also $[3,8,19,22])$. In the sequel we will provide a proof of these inequalities on the grounds of Theorem 3.3, and discuss the equality signs in (6.1). As in Theorem 3.3, the only assumption on $\Omega$ is to be a simply connected Lipschitz domain.

We start from the representations (3.5) and (3.10) of the diagonal elements of the polarization tensor, and first note that

$$
\int_{-\infty}^{\infty} \mathrm{d} \alpha_{\lambda}=|\Omega|
$$

by virtue of (3.15). From (3.5) and (3.10) we further conclude that the trace of $M(\mu ; \Omega)$ is given by

$$
\operatorname{Tr}(M(\mu ; \Omega))=\int_{-\infty}^{\infty} \frac{2 \mu}{\mu^{2}-\lambda^{2}} \mathrm{~d} \alpha_{\lambda} .
$$

Since $\alpha \ll \alpha^{\text {symm }}$ (cf. (3.14)), and since the support of $\alpha^{\text {symm }}$ is contained in $(-1 / 2,1 / 2)$ by virtue of Theorem 3.3 , it follows that

$$
\frac{2}{|\mu|} \int_{-\infty}^{\infty} \mathrm{d} \alpha_{\lambda} \leq|\operatorname{Tr}(M(\mu ; \Omega))|<\frac{2|\mu|}{\mu^{2}-1 / 4} \int_{-\infty}^{\infty} \mathrm{d} \alpha_{\lambda}
$$

for every $\mu \geq 1 / 2$, and the same argument applies to values of $\mu$ less or equal than $-1 / 2$. Making use of (6.2) we thus obtain the first isoperimetric inequality (6.1a).

The above derivation reveals that the lower bound in (6.1a) is attained for any value of $\mu$ with $|\mu| \geq 1 / 2$, if and only if $\alpha$ is supported in the origin, that is, if $M(\mu ; \Omega)$ is a rational function with a simple pole at $\lambda=0$. As we have discussed in Section 5, this is the case, if and only if $\Omega$ is a disk; this is known as a conjecture of Pólya and Szegö. In contrast, the upper bound is never attained: that the upper bound cannot be attained by domains $\Omega$ with a certain "thickness" has been shown by Capdeboscq and Kang [6].

Concerning the trace of $M\left(\mu_{*} ; \Omega\right)^{-1}$ for a given $\mu_{*} \in \mathbb{R} \backslash(-1 / 2,1 / 2)$, we first rotate the coordinate system in such a way that the orthogonal eigenbasis of (the real symmetric matrix) $M\left(\mu_{*} ; \Omega\right)$ is parallel to the two coordinate axes. This means that the off-diagonal entries of the polarization tensor (in the rotated coordinate system) have a root at $\mu=\mu_{*}$, and hence,

$$
\operatorname{Tr}\left(M\left(\mu_{*} ; \Omega\right)^{-1}\right)=\frac{1}{f_{+}\left(\mu_{*}\right)}+\frac{1}{f_{-}\left(\mu_{*}\right)},
$$

where

$$
f_{ \pm}(\mu)=\int_{-\infty}^{\infty} \frac{1}{\mu \pm \lambda} \mathrm{d} \alpha_{\lambda}
$$


are the diagonal entries (3.5) and (3.10) of the polarization tensor in the rotated coordinates. Straightforward differentiation reveals that $\left(1 / f_{ \pm}\right)^{\prime}(\mu)>0$ for $\mu \geq 1 / 2$ and

$$
\begin{aligned}
\left(\frac{1}{f_{ \pm}}\right)^{\prime \prime}(\mu)=2 & \left(\int_{-\infty}^{\infty} \frac{1}{\mu \pm \lambda} \mathrm{d} \alpha_{\lambda}\right)^{-3} . \\
& \left\{\left(\int_{-\infty}^{\infty} \frac{1}{(\mu \pm \lambda)^{2}} \mathrm{~d} \alpha_{\lambda}\right)^{2}-\int_{-\infty}^{\infty} \frac{1}{(\mu \pm \lambda)^{3}} \mathrm{~d} \alpha_{\lambda} \int_{-\infty}^{\infty} \frac{1}{\mu \pm \lambda} \mathrm{d} \alpha_{\lambda}\right\},
\end{aligned}
$$

where the term in curled braces on the right is nonpositive by virtue of the CauchySchwarz inequality; to be more precise, the second derivative is strictly negative, unless the measure $\alpha$ is concentrated on one single point in the interval $(-1 / 2,1 / 2)$.

It follows that

$$
g(\mu)=\frac{1}{f_{+}(\mu)}+\frac{1}{f_{-}(\mu)}
$$

is concave for $\mu \geq 1 / 2$. Moreover, in view of (6.2) there holds

$$
\frac{1}{f_{ \pm}(\mu)}=\frac{\mu}{|\Omega|} \pm \frac{1}{|\Omega|^{2}} \int_{-\infty}^{\infty} \lambda \mathrm{d} \alpha_{\lambda}+O\left(\mu^{-1}\right), \quad \mu \rightarrow \infty
$$

and hence,

$$
g(\mu)=\frac{2 \mu}{|\Omega|}+O\left(\mu^{-1}\right), \quad \mu \rightarrow \infty .
$$

Since $g$ is concave this implies that

$$
g(\mu) \leq \frac{2 \mu}{|\Omega|} \quad \text { for all } \mu \geq 1 / 2,
$$

with equality for any single value of $\mu$, if and only if $\alpha$ is concentrated on a single point $\lambda \in(-1 / 2,1 / 2)$. If this happens to be the case then the polarization tensor has the representation

$$
M(\mu ; \Omega)=\left[\begin{array}{cc}
\frac{r^{2}}{\mu-\lambda} & 0 \\
0 & \frac{r^{2}}{\mu+\lambda}
\end{array}\right] \quad \text { for all } \mu \in \mathbb{C}
$$

in the rotated coordinate system, and hence, $\Omega$ is an ellipse (or a circle, when $\lambda=0$ ), as we have seen in Section 5. Note that (6.3) immediately implies that

$$
|g(\mu)| \leq \frac{2|\mu|}{|\Omega|} \quad \text { for all } \mu \in \mathbb{R} \backslash(-1 / 2,1 / 2),
$$

because $f_{+}(-\mu)=-f_{-}(\mu)$ for every $\mu \in \mathbb{C}($ cf. (3.10)).

We thus have established the other isoperimetric inequality (6.1b), and have shown that equality holds for any value of $\mu \in \mathbb{R} \backslash(-1 / 2,1 / 2)$, if and only if $\Omega$ is an ellipse; the latter was proved first by Kang and Milton $[15,16]$. 
7. Concluding remarks. We have derived an analytic representation of polarization tensors as function of the admittivity contrast in terms of the spectral decomposition of the double layer integral operator associated with the underlying domain. Since our arguments rely on complex analysis and layer potential techniques, a generalization of this result to three-dimensional objects or more complicated nonconstant conductivity inhomogeneities (see, e.g., Capdeboscq and Vogelius [7]) is not straightforward.

We have considered two applications of this analytic representation, namely (i) an elementary proof of the Hashin-Shtrikman bounds for the trace of polarization tensors and their inverses in terms of the area of the associated domain, and (ii) we have established a one-to-one correspondence between ellipses and certain polarization tensors with at most two poles as a function of the admittivity contrast. It remains an open problem to what extent the shape of a general planar simply connected and bounded Lipschitz domain is determined by the polarization tensor as a function of the admittivity contrast.

Finally we note that the results of this work have further been utilized in [12] to analyze a multi-frequency MUSIC-type method for electrical impedance tomography.

Acknowledgements. We like to thank Grame W. Milton (University of Utah) for pointing out to us relevant literature from the theory of composite materials.

\section{REFERENCES}

[1] H. Ammari, Y.T. Chow, K. Liu, and J. Zou, Optimal shape design by partial spectral data, Preprint, arXiv:1310.6098v1 [math.OC] (2013).

[2] H. Ammari and H. Kang, Polarization and Moment Tensors with Applications to Inverse Problems and Effective Medium Theory, Springer, New York, 2007.

[3] A.Y. Belyaev and S.M. Kozlow, Hierarchical structures and estimates for homogenized coefficients, Russian J. Math. Phys. 1 (1993), pp. 5-18.

[4] D.J. Bergman, The dielectric constant of a composite material - a problem in classical physics, Phys. Rep. 43 (1987), pp. 377-407

[5] M. Brühl, M. Hanke, And M.S. Vogelius, A direct impedance tomography algorithm for locating small inhomogeneities, Numer. Math. 93 (2003), pp. 635-654.

[6] Y. CAPDEBosca AND H. Kang, Improved bounds on the polarization tensor of thick domains, in Inverse Problems, Multi-Scale Analysis, and Effective Medium Theory, H. Ammari and H. Kang, eds., Contemp. Math., Amer. Math. Soc., Providence, RI, 2006, pp. 69-74.

[7] Y. CapdeboscQ and M.S. Vogelius, A general representation formula for boundary voltage perturbations caused by internal conductivity inhomogeneities of low volume fraction, M2AN Math. Model. Numer. Anal. 37 (2003), pp. 159-173.

[8] Y. CAPdeboscQ And M.S. Vogelius, Pointwise polarization tensor bounds, and applications to voltage perturbations caused by thin inhomogeneities, Asymptot. Anal. 50 (2006), pp. 175204.

[9] D.J. Cedio-Fengya, S. Moskow, and M.S. Vogelius, Identification of conductivity imperfections of small diameter by boundary measurements. Continuous dependence and computational reconstruction, Inverse Problems 14 (2001), pp. 553-595.

[10] L.C. Evans And R.F. Gariepy, Measure Theory and Fine Properties of Functions, CRC Press, Boca Raton, 1992.

[11] K. Golden and G. Papanicolaou, Bounds for effective parameters of heterogeneous media by analytic continuation, Comm. Math. Phys. 90 (1983), pp. 473-491.

[12] R. Griesmaier and M. Hanke, Multifrequency impedance imaging with multiple signal classification, SIAM J. Imaging Sci. 8 (2015), pp. 939-967.

[13] Z. Hashin And S-A. Shtrikman, A variational approach to the theory of the elastic behaviour of multiphase materials, J. Mech. Phys. Solids 11 (1963), pp. 127-140.

[14] J. Helsing and K-M. Perfekt, On the polarizability and capacitance of the cube, Appl. Comput. Harmonic Anal. 34 (2013), pp. 445-468. 
[15] H. Kang and G.W. Milton, On conjectures of Polya-Szegő and Eshelby, in Inverse Problems, Multi-Scale Analysis, and Effective Medium Theory, H. Ammari and H. Kang, eds., Contemp. Math., Amer. Math. Soc., Providence, RI, 2006, pp. 75-79.

[16] H. Kang And G.W. Milton, Solutions to the Pólya-Szegő conjecture and the weak Eshelby conjecture, Arch. Ration. Mech. Anal. 188 (2008), pp. 93-116.

[17] J.B. Keller, A theorem on the conductivity of a composite medium, J. Math. Phys. 5 (1964), pp. $548-549$.

[18] D. Khavinson, M. Putinar, and H.S. Shapiro, Poincaré's variational problem in potential theory, Arch. Ration. Mech. Anal. 185 (2007), pp. 143-184.

[19] R.V. Kohn and G.W. Mitton, On bounding the effective conductivity of anisotropic composites, in Homogenization and Effective Moduli of Materials and Media, J.L. Ericksen, D. Kinderlehrer, R.V. Kohn, and J.-L. Lions, eds., Springer, New York, 1986, pp. 97-125.

20] R. Kress, Linear Integral Equations, 3rd ed., Springer, New York, 2014.

[21] N.S. Landkof, Foundations of Modern Potential Theory, Springer, Berlin, 1972.

[22] R. Lipton, Inequalities for electric and elastic polarization tensors with applications to random composites, J. Mech. Phys. Solids 41 (1993), pp. 809-833.

[23] L.P. Liu, Solutions to the Eshelby conjectures, Proc. Roy. Soc. London Ser. A 464 (2008), pp. 573-594.

[24] W. McLean, Strongly Elliptic Systems and Boundary Integral Equations, Cambridge University Press, Cambridge, 2000.

[25] G.W. Milton, The Theory of Composites, Cambridge University Press, Cambridge, 2002.

[26] J. Plemelu, Potentialtheoretische Untersuchungen, B.G. Teubner, Leipzig, 1911.

[27] C.Q. Ru and P. Schiavone, On the elliptic inclusion in anti-plane shear, Math. Mech. Solids 1 (1996), pp. 327-333

[28] W. Rudin, Functional Analysis, 2nd Ed. McGraw-Hill, New York, 1991.

[29] M. Schiffer, The Fredholm eigen values of plane domains, Pacific J. Math. 7 (1957), pp. 11871225 .

[30] M. Schiffer, Fredholm eigenvalues and conformal mapping, in G. Fichera, ed., Autovalori e autosoluzioni, Berlin, Heidelberg, Springer, 2011, pp. 205-234.

[31] O. Steinbach and W.L. Wendland, On C. Neumann's method for second-order elliptic systems in domains with non-smooth boundaries, J. Math. Anal. Appl. 262 (2001), pp. 733748.

[32] G. Verchota, Layer potentials and regularity for the Dirichlet problem for Laplace's equation in Lipschitz domains, J. Funct. Anal. 59 (1984), pp. 572-611. 